\title{
Retraction Note to: Calcium/calmodulin-dependent kinase inhibitor induces growth inhibition, cell cycle arrest, and apoptosis in human choriocarcinoma cells
}

\author{
Noriyuki Takai $^{1} \cdot$ Tami Ueda $^{1} \cdot$ Naoko Kira $^{1} \cdot$ Terukazu Ishii $^{1} \cdot$ Toshie Yoshida $^{1}$. \\ Satoko Koga ${ }^{1} \cdot$ Masakazu Nishida $^{1} \cdot$ Kaei Nasu $^{1} \cdot$ Hisashi Narahara ${ }^{1}$
}

Published online: 21 June 2015

(C) International Society of Oncology and BioMarkers (ISOBM) 2015

Retraction to: Tumor Biol. (2012) 33:1053-1058

DOI 10.1007/s13277-012-0339-x

This article has been retracted on request by the corresponding author. The authors have identified errors affecting several figure panels in which original data were processed inappropriately such that the panels in Fig. 4: "Expression of the cell cycle- and apoptosis-related proteins treated with KN-93 in choriocarcinoma cells measured by Western blot analysis. Choriocarcinoma cells were treated with KN-93, and cell lysates were harvested after $48 \mathrm{~h}$. Western blot analysis was performed with a series of antibodies (cyclin A, cyclin D1, Bcl-2, and cleaved caspase-3). Control cells were treated with vehicle alone. The amount of protein was normalized by comparison to levels of GAPDH" do not accurately report the original data. The other authors were not involved in making these figures. The corresponding author, Dr. Takai, sincerely apologizes to the co-authors and the scientific community for any inconvenience that this might cause.

The online version of the original article can be found at http://dx.doi.org/ 10.1007/s13277-012-0339-x.

Noriyuki Takai

takai@oita-u.ac.jp

Department of Obstetrics and Gynecology, Faculty of Medicine, Oita University, 1-1 Idaigaoka, Hasama-machi, Yufu-shi, Oita 879-5593, Japan 